\title{
SH1-dependent maize seed development and starch synthesis via modulating carbohydrate flow and osmotic potential balance
}

Ke Zhang ${ }^{1+}$, Li Guo ${ }^{1 \dagger}$, Wen Cheng ${ }^{2+}$, Baiyu Liu' ${ }^{1}$ Wendi Li ${ }^{1}$, Fei Wang ${ }^{3}$, Changzheng Xu ${ }^{4}$, Xiangyu Zhao ${ }^{5}$, Zhaohua Ding ${ }^{2}$, Kewei Zhang ${ }^{1}$ and Kunpeng $\mathrm{Li}^{1 *}$ (D)

\begin{abstract}
Background: As the main form of photoassimilates transported from vegetative tissues to the reproductive organs, sucrose and its degradation products are crucial for cell fate determination and development of maize kernels. Despite the relevance of sucrose synthase SH1 (shrunken 1)-mediated release of hexoses for kernel development, the underlying physiological and molecular mechanisms are not yet well understood in maize (Zea mays).

Results: Here, we identified a new allelic mutant of SH1 generated by EMS mutagenesis, designated as sh ${ }^{*}$. The mutation of $\mathrm{SH} 1$ caused more than $90 \%$ loss of sucrose synthase activity in $s h{ }^{*}$ endosperm, which resulted in a significant reduction in starch contents while a dramatic increase in soluble sugars. As a result, an extremely high osmolality in endosperm cells of $s h{ }^{*}$ was generated, which caused kernel swelling and affected the seed development. Quantitative measurement of phosphorylated sugars showed that Glc-1-P in endosperm of sh ${ }^{*}(17$ $\mu \mathrm{g} \mathrm{\textrm {g } ^ { - 1 }} \mathrm{FW}$ ) was only $5.2 \%$ of that of wild-type $\left(326 \mu \mathrm{g} \mathrm{g}{ }^{-1} \mathrm{FW}\right)$. As a direct source of starch synthesis, the decrease of Glc-1-P may cause a significant reduction in carbohydrates that flow to starch synthesis, ultimately contributing to the defects in starch granule development and reduction of starch content.

Conclusions: Our results demonstrated that SH1-mediated sucrose degradation is critical for maize kernel development and starch synthesis by regulating the flow of carbohydrates and maintaining the balance of osmotic potential.
\end{abstract}

Keywords: Glucose-1-phosphate, Seed development, SH1, Soluble sugars, Starch synthesis, Zea mays

\footnotetext{
* Correspondence: likp@sdu.edu.cn

${ }^{\dagger}$ Ke Zhang, Li Guo and Wen Cheng contributed equally to this work.

${ }^{1}$ The Key Laboratory of Plant Development and Environment Adaptation

Biology, Ministry of Education, School of Life Science, Shandong University,

Qingdao 266237, China

Full list of author information is available at the end of the article
}

(c) The Author(s). 2020 Open Access This article is licensed under a Creative Commons Attribution 4.0 International License, which permits use, sharing, adaptation, distribution and reproduction in any medium or format, as long as you give appropriate credit to the original author(s) and the source, provide a link to the Creative Commons licence, and indicate if changes were made. The images or other third party material in this article are included in the article's Creative Commons licence, unless indicated otherwise in a credit line to the material. If material is not included in the article's Creative Commons licence and your intended use is not permitted by statutory regulation or exceeds the permitted use, you will need to obtain permission directly from the copyright holder. To view a copy of this licence, visit http://creativecommons.org/licenses/by/4.0/ The Creative Commons Public Domain Dedication waiver (http://creativecommons.org/publicdomain/zero/1.0/) applies to the data made available in this article, unless otherwise stated in a credit line to the data. 


\section{Background}

Maize kernel development is a dynamic and sophisticated developmental process orchestrated by complicated regulatory networks involving a large number of genes. Owing to molecular and genetics studies, many genes that are essential for kernel development have been identified, including those involved in basal endosperm transfer layer (BETL) differentiation [1-3], sugar transportation [4-7], sucrose metabolism and starch synthesis [8-12].

As a major organ for nutrition storage, kernels assimilate a large amount of carbohydrates that are produced in photosynthetic cells. Apart from energy supply, most of those carbohydrates are converted into starch, which accounts for $70-75 \%$ of seed weight $[13,14]$. Sucrose is the main form of photoassimilates that is transported from photosynthetic cells to kernels [4-6, 15]. The families of sucrose transporters (SUTs) and SWEET proteins, have been shown to participate in sucrose transportation [7]. SUTs are the major sucrose transporters [7], while SWEETs constitute a new hexose and sucrose transporters family. In maize, $Z m S W E E T 4 c$ is mainly expressed in the BETL, the entry point of sucrose into seed, and is thereby critical for BETL formation and seed filling [1]. During seed development, sucrose is not only the raw material for cell wall formation, starch synthesis and glycolysis, but also an important signaling regulator of hormonal signaling and cell fate determination by affecting the expressions of related genes $[12,16]$. Several studies have shown that the expression of multiple key genes, such as ZmMRP-1, ZmYUC1, cell wall invertase MINIATURE1 $(M n 1)$ and $Z m S W E E T 4 c$, is modulated by the content of sugars including sucrose and its degradation products during early kernel development, and function in regulation of BETL cells differentiation and kernel development $[1,2]$.

Sucrose catabolism mainly depends on two enzyme families: invertase (INV) and sucrose synthase (SUS) [16-19]. INVs catalyze the irreversible decomposition of sucrose into glucose and fructose, which are the substrates of glucose-6-phosphate (Glc-6-P) and fructose-6-phosphate (Fru-6-P), the important precursors for multiple downstream pathways [20]. Six invertases have been annotated in maize genome so far [21]. Among them, INCW2 encoded by $M n 1$ has been identified as a BETL-specific protein [15, 22-24]. The loss-of-function of $M n 1$ resulted in higher sucrose content and lower hexose to sucrose ratio in endosperm cells, and $70 \%$ loss of seeds weight [22, 24]. Gene expression analysis indicated several key genes involved in starch synthesis (Sh2 and Bt2) and encoding sucrose synthase (SH1, SUS1 and SUS2) were significantly down-regulated in the $m n 1$ mutant [24]. These data indicated that abnormal sucrose metabolism caused by $M n 1$ mutation led to differential expression of a large number of genes related to carbon metabolism, which in turn affected maize seed development and yield.
In contrast with INVs, functional characterization of sucrose synthase is relatively lacking during seed development in maize. SUSs can reversibly transform sucrose into fructose and uridine-diphosphoglucose (UDP-Glc). UDPGlc acts as a substrate for cellulose synthesis, the concentration of which can affect cell wall formation. In addition, UDP-Glc can be converted into Glc-1-P, a carbon source for starch synthesis, which is catalyzed by UDP-glucose pyrophosphorylase. Therefore, SUSs are considered to play important roles in cell wall formation and starch synthesis [16-18]. In maize genome, 20 genes were predicted to encode sucrose synthase, three of which including SUS1, SUS2, and SH1 have been functionally identified [25]. SUS2 encoding a 'housekeeping' SUS isozyme is localized in the cytoplasm and cleaves sucrose for cytoplasmic metabolism [26]. SH1 and SUS1 encode two biochemically similar isozymes [27]. Both of them, unlike SUS2, were proven to be associated with membranes, implying their distinct functions from SUS2 $[9,26]$. Previous studies have shown that the loss-of-function of $\mathrm{SH} 1$ resulted in a significant reduction of sucrose synthase activity and a decreased starch accumulation, thereby leading to shrunken kernels [28]. The starch contents in kernels of sh1 SUS1 and sh1 sus1-1 genotypes are 78 and 53\% of SH1 SUS1, respectively. Chourey et al. (1998) deemed that SH1 and SUS1 predominately functioned in cellulose biosynthesis and starch biosynthesis, respectively [8, 27]. The functional loss of $\mathrm{SH} 1$ caused the restriction of UDP-Glc into cellulose biosynthesis during cell elongation [8]. A recent study confirmed that $S H 1$ also played an important role in starch synthesis, and $S H 1$ null mutation resulted in a significant increase in the ratio of amylose to amylopectin in the endosperm [11]. These above studies provided a preliminary understanding of the function of $\mathrm{SH} 1$ in maize seed development. However, the roles of sucrose degradation pathway catalyzed by $\mathrm{SH} 1$ in maintenance of carbon metabolism balance and regulation of gene expression during seed development are not yet fully understood.

In the present study, we revealed the roles of $\mathrm{SH} 1$ in appropriate carbon partitioning, maintaining the balance of osmotic potential, regulating the starch synthesis and seed development via characterization of $s h 1 *$ mutant. Null mutation of SH1 led to less carbohydrates flowing to starch synthesis pathway. A large number of carbohydrates exist in the form of soluble sugars. The carbon metabolic disorder induced by $S H 1$ mutation leads to the kernel development arrest and shrunken phenotype in the $\operatorname{sh} 1 *$ mutant.

\section{Results}

sh 1 * produces shrunken kernels with reduced starch contents and dysplastic starch granules

The $s h 1 *$ mutant was obtained by ethyl methanesulfonate (EMS) mutagenesis. It was crossed with W64A to 
produce an F2 genetically-isolated population that displayed a 3:1 segregation of wide-type $(+/+$ and $\operatorname{sh} 1 * /+)$ and shrunken $(\operatorname{sh} 1 \% / \operatorname{sh} 1 *)$ phenotypes (Fig. 1a). Compared with Z58, kernels of the $s h 1^{*}$ were badly concave in the middle of the top after dehydration, and had less cytoplasmic dense inside of the kernels (Fig. 1a, b). The 100 -seed weight of $s h 1 *$ was about $33 \%$ less than that of Z58 (Fig. 1c). To explore the effects of the developmental

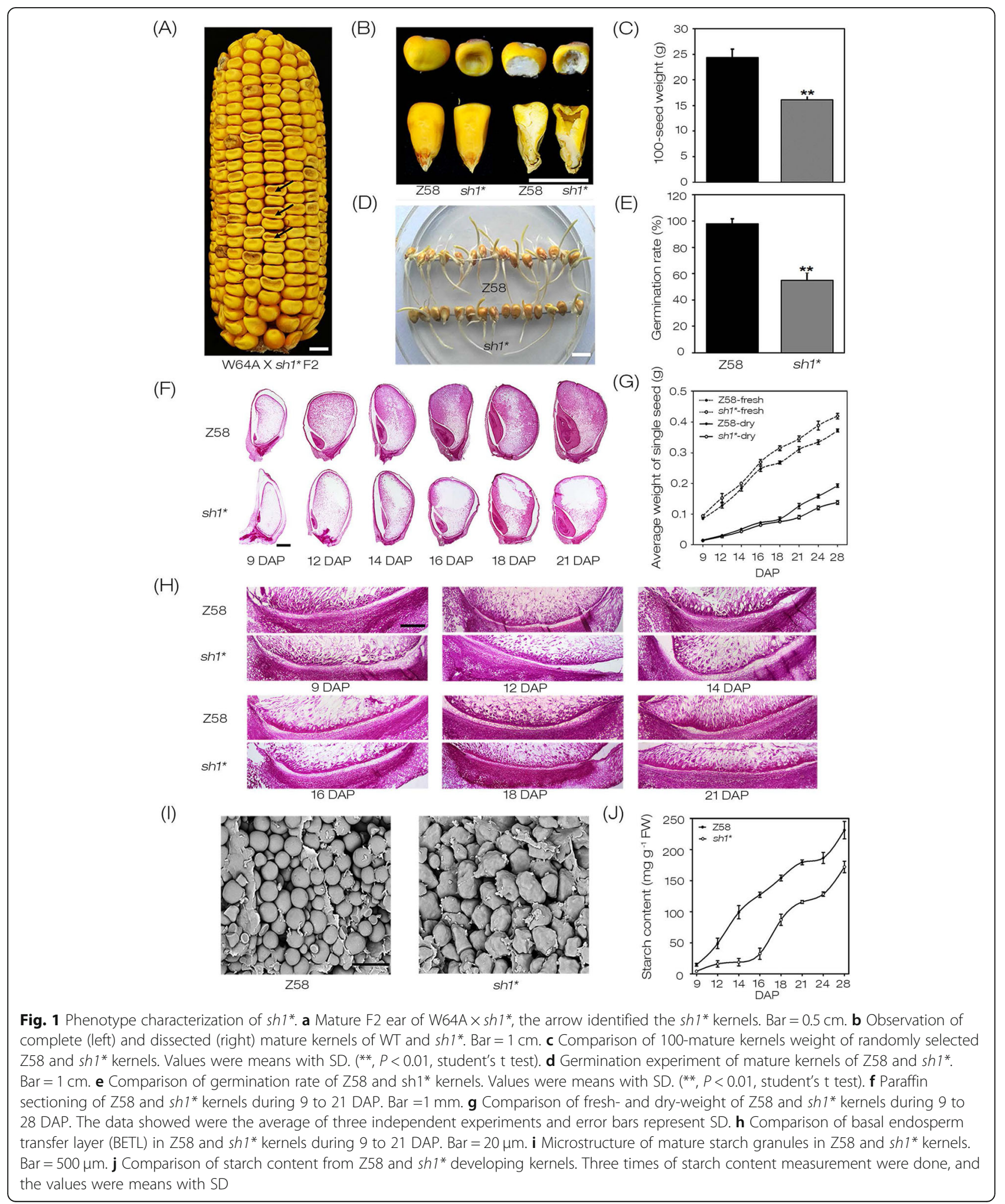


defects on seed germination, germination experiments of sh $1 *$ and Z58 were performed on 1/2 MS medium. Our results showed that the germination rate of $s h 1$ * seeds was only about $54 \%$ of that in Z58 (Fig. 1d, e).

To investigate the differences in kernels development between $s h 1^{*}$ and Z58, their kernels at 9, 12, 14, 16, 18 and 21 days after pollination (DAP) were observed. Longitudinal sections indicated that the kernel development of $\operatorname{sh} 1^{*}$ were significantly delayed compared with Z58. The embryos were smaller and the endosperm had dramatically less materials accumulation in $s h 1^{*}$ at same stages than Z58 (Fig. 1f). The cell deficiency in endosperm of $s h 1^{*}$ at $12,14,16,18$ and 21 DAP were observed in the centrally-located starch storage cells, except for the kernels of 9 DAP (Fig. 1f). Detection of the fresh- and dry-weight of kernels indicated that there were no significant differences between $s h 1^{*}$ and Z58 at 9, $12,14,16$ DAP, whereas they began to show difference after 18 DAP that became more and more notable later on during the developmental process (Fig. 1g). Although the dry-weight of $s h 1^{*}$ kernels was significantly lower than that of Z58 at the same stage after 18 DAP, it had obviously higher fresh weights. These results indicated that, compared to Z58, sh1* kernels were able to absorb more water, but accumulate fewer dry contents. As sink organs, developing kernels obtain nutrients and storage substances from vegetative tissues, and this process relies on BETL cells. Our results indicated that at the same stages, the BETL development of $s h 1^{*}$ kernels was relatively delayed compared to WT (Fig. 1h), which might affect the accumulation of dry matter in $s h 1^{*}$ kernels.

Observation of sh1* and Z58 mature kernels indicated that starch granules in endosperm of Z58 kernels exhibited a regular round shape and densely distributed, whereas those of $\operatorname{sh} 1 *$ were of irregular shape and loosely arranged (Fig. 1i). The content of starch in $s h 1^{*}$ kernels was significantly lower than that of Z58 (Fig. 1j). These results indicated that the development of kernels was weakened and the accumulation of starch was reduced in $\operatorname{sh} 1 \%$.

\section{$\mathrm{Sh} 1 *$ encodes the sucrose synthase $\mathrm{SH} 1$}

Genetic fine-mapping of $s h 1$ * was performed with 2400 shrunken kernels from F2 mapping population, and the sh1* locus was localized in a region of $97.12 \mathrm{~kb}$ on chromosome 9 between the self-created molecular markers ZM0064 and ZM0059 (Fig. 2a). Retrieving the

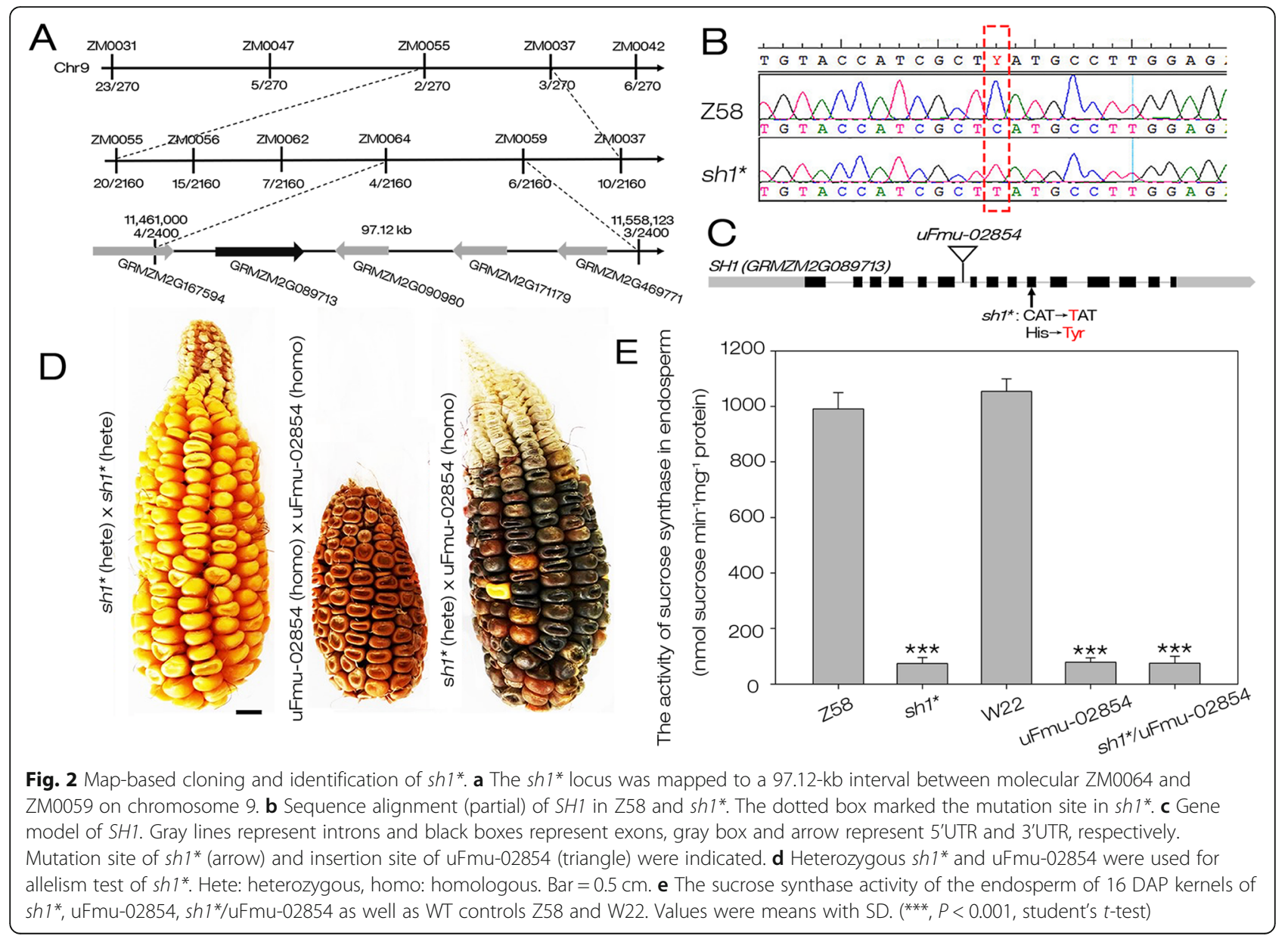


maizeGDB database (https://www.maizegdb.org/), we found that there are 5 predicted genes within this interval, including GRMZM2G167594, GRMZM2G089713, GRMZM2G090980, GRMZM2G171179 and GRMZM2G469771 (Fig. 2a). Nucleotide sequence analysis of $\mathrm{Z} 58$ and $s h 1 *$ revealed a $\mathrm{C}$ to $\mathrm{T}$ substitution at $1297 \mathrm{bp}$ (the "A" of the translation start codon "ATG" was designated "+ 1") in open reading frame (ORF) of GRMZM2G089713 (Fig. 2b and Fig. S1), caused a mutation of histidine to tyrosine (Fig. 2c). The ORF sequences of GRMZM2G167594, GRMZM2G090980, GRMZM2G171179 and GRMZM2G469771 have no differences between Z58 and sh1*. Therefore, GRMZM2G089713 should be the best candidate gene for the Sh1* locus.

To confirm GRMZM2G089713 is the causative gene for the $s h 1 *$ phenotype, an allelism test was performed by crossing sh1* with uFmu-02854, a UniformMu insertion mutant of GRMZM2G089713 with W22 genetic background (Fig. 2c). The uFmu-02854 (homozygous) was crossed to $\operatorname{sh} 1 *$ (heterozygous), and the F1 ears displayed a 1:1 segregation of WT (+/uFmu-02854) and shrunken $(s h 1 \% /$ uFmu-02854) phenotype (Fig. 2d), indicating the uFmu-02854 cannot complement sh1* Therefore, GRMZM2G089713 was the causative gene for the sh1* mutation, and encoded a sucrose synthase SH1 [28]. To further investigate the effect of a single base mutation of $S H 1$ on its enzymatic activity in the sh 1 * mutant, we tested the sucrose synthase activity of the endosperm of 16 DAP kernels of $s h 1^{*}$, uFmu-02854, sh1\%/uFmu-02854 as well as WT controls Z58 and W22. The results showed that sucrose synthase activity decreased by approximately 9193\% in the endosperm of $s h 1 *$, uFmu-02854 and sh1*/ uFmu-02854, compared with WT (Fig. 2e). Therefore, we believed that the mutation in $s h 1 *$ caused its severe loss of SH1 function, even inactivation.

\section{SH1 displayed a close phylogenetic relationship with} SUS1 whereas distinct tissue-specific expression patterns To investigate the phylogenetic relationship of $\mathrm{SH} 1$ and its 19 homologs in maize, we constructed a phylogenetic tree based on their amino acids sequences (Fig. 3a). The result showed that SH1 was highly close to SUS1 (GRMZM2G152908). Multiple sequence alignment of SH1, SUS1 and SUS2 (GRMZM2G318780), three sucrose synthases had been identified in maize so far $[26,27]$, revealing the high identity and conservation of their amino acids sequences, especially SH1 and SUS1 (Fig. 3b). These results suggested that the two of them, especially $\mathrm{SH} 1$ and SUS1, might share similar biological functions. However,

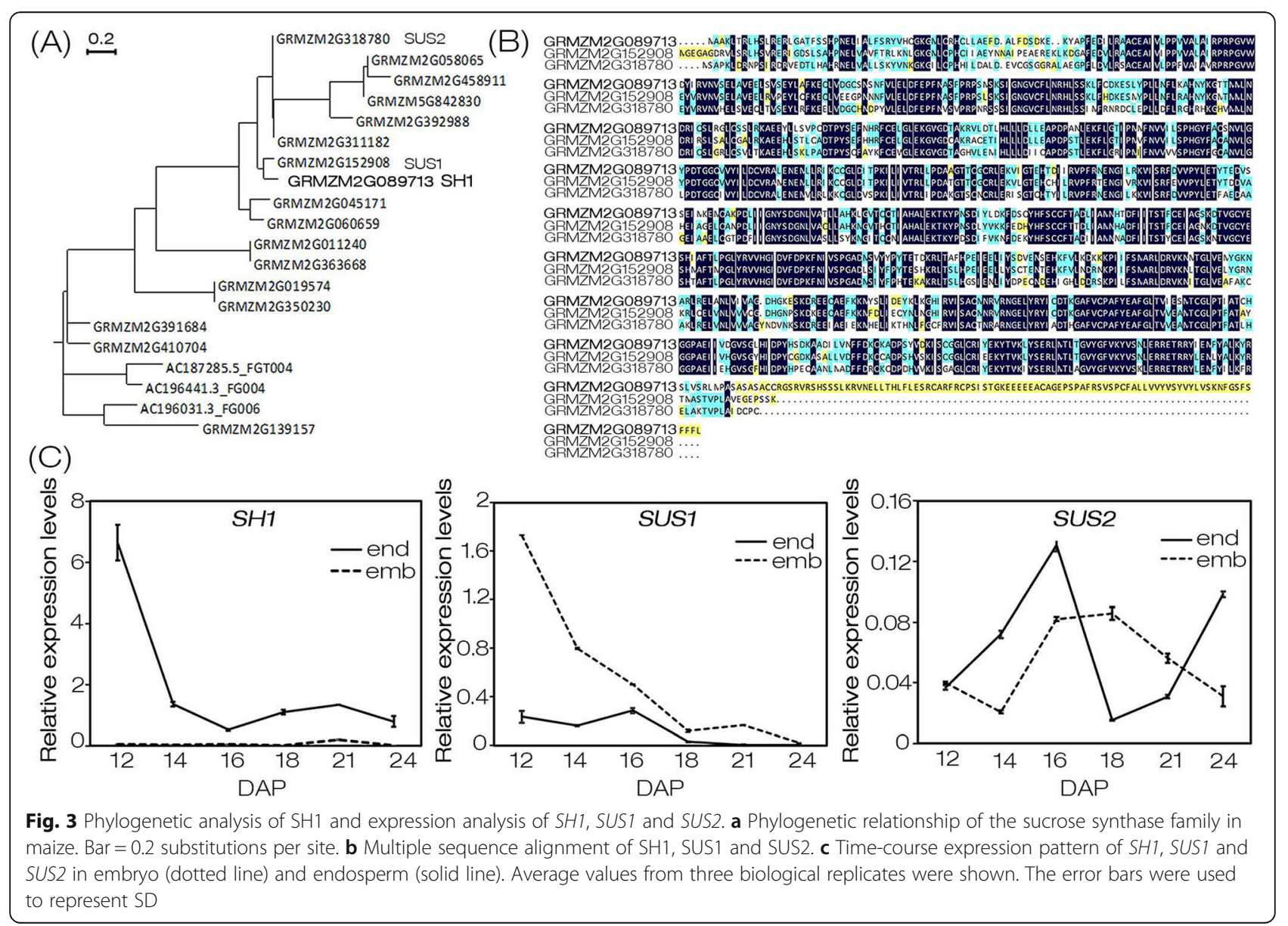


SUS1 and SUS2 were not able to complement the functional deletion mutation of $S H 1$ in $s h 1 \%$. To investigate this reason, time-course expression patterns analyses of $\mathrm{SH}$, SUS1 and SUS2 in embryo and endosperm of maize were determined. Our results indicated that $S H 1$ was primarily expressed in endosperm while the SUS1 transcripts mainly accumulated in the embryo. SUS2 was expressed in both embryo and endosperm in a different expression trend from with SH1 and SUS1, and its expression levels in the embryo and endosperm were around $1 / 10$ or less than that of SH1 (Fig. 3c). In addition, previous studies had shown that SH1 and SUS2 displayed different subcellular localizations $[9,26,27]$. These results suggested that their differential expression might account for the inability of SUS1 and SUS2 to complement SH1 function.

\section{The distribution of SH1 was mostly associated with membranes}

Subbaiah and Sachs (2001) found that SUSs were mainly distributed in the soluble fraction, and only a few of them were membrane-associated revealed by protein separation and immunoblot analysis using non-specific antibody [9]. Duncan et al. (2006) showed that SH1 was associated with membranes, but it was primarily distributed in soluble fraction by protein extraction and immunoblot analysis using isoform-specific antibody [26]. The accuracy of these results largely depended on protein extraction process and antibody specificity. To clarify the localization of SH1, we constructed the 35S::SH1:GFP plasmid and introduced it together with a membrane protein marker vector CD3-1001 (positive control) into tobacco epidermal cells (Fig. 4a) and protoplasts (Fig. 4b). Laser confocal microscopy revealed that the GFP signals displayed overlapping pattern with the membrane protein marker. This result indicated that the distribution of $\mathrm{SH} 1$ may be mostly associated with membranes.

sh 1 * affected the balance of carbon metabolism and the maintenance of osmolality during kernel development When harvesting F2 ears of W64A $\times \operatorname{sh} 1 *$, we found that mutant kernels $(\operatorname{sh} 1 \% \operatorname{sh} 1 *)$ were swollen extremely and appeared to be bursting (Fig. 5a). This phenotype suggested that the mutant kernels might possess a higher osmotic potential, which drives water absorption in kernels. To clarify the assumption, the osmotic potential of sh1* and Z58 kernels at 9, 12, 14, 16, 18, 21, 24, 28 DAP were detected. Except the kernels at 9 and 28 DAP, the sh $1^{*}$ kernels had a significantly higher osmotic potential than that of Z58. In particular, the osmolality in $s h 1^{*}$ kernels at 14, 16 and 18 DAP were more than twice of that in Z58 (Fig. 5b). These results were consistent with that the $s h 1 *$ kernels had less dry matter accumulation but higher fresh weight than those of Z58 (Fig. 1g).

As a sucrose synthase, $\mathrm{SH} 1$ is mainly responsible for the degradation of sucrose. The loss-of-function of SH1 may affect the composition and content of carbohydrates, leading to a change in osmotic potential. Our results showed that the total soluble sugars in $s h 1^{*}$ kernels at $9,12,14,16,18,21,24$ and 28 DAP were significantly higher than that of Z58, especially for $\operatorname{sh} 1^{*}$ kernels of 14

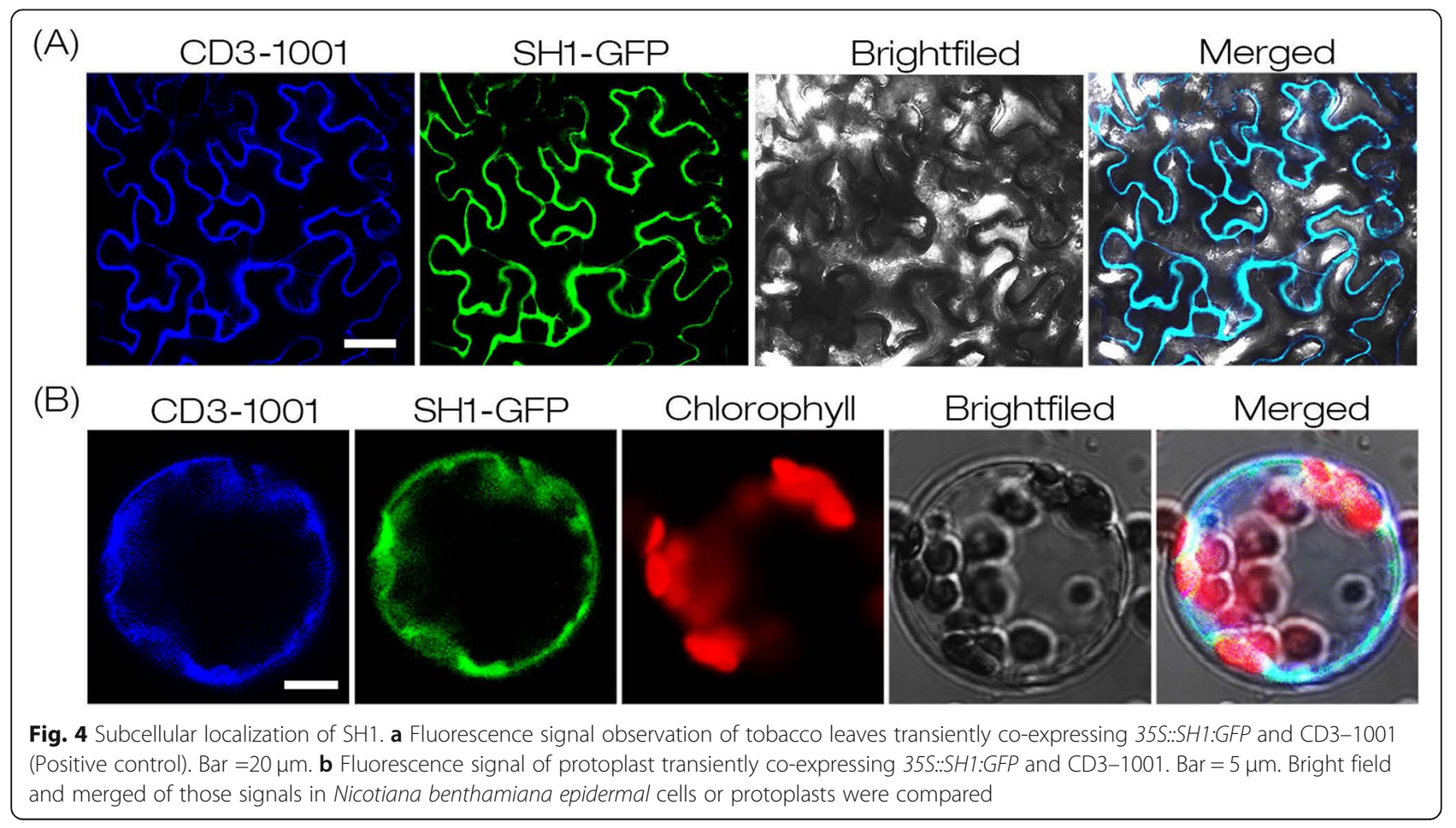



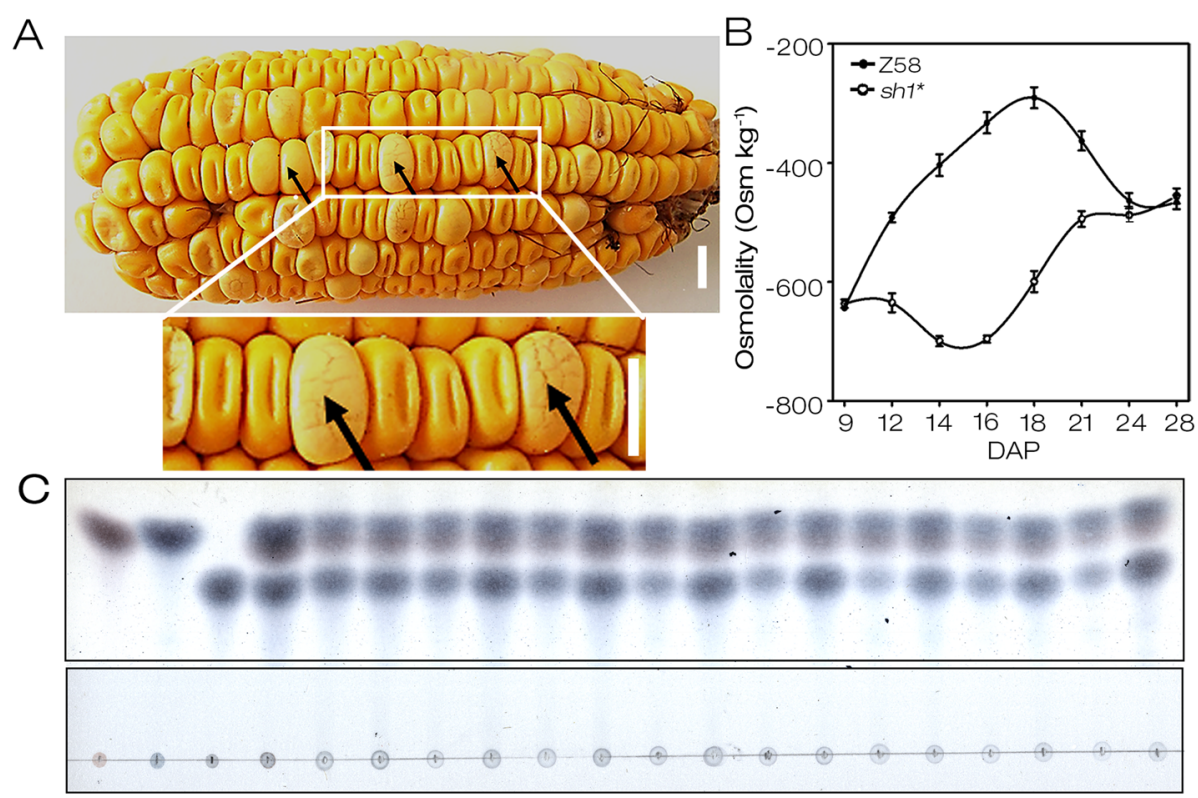

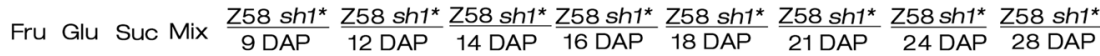
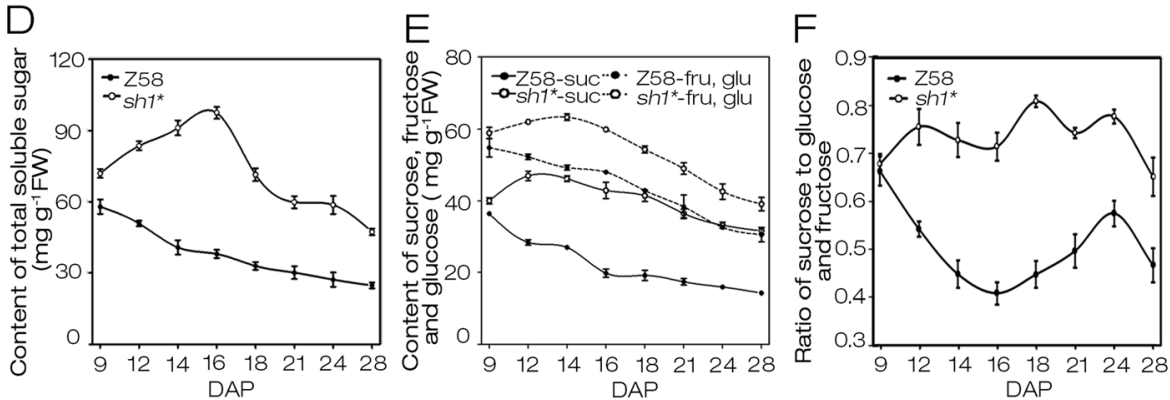

Fig. 5 Osmolality and soluble sugar contents in $s h 1^{*}$. a A freshly harvested F2 ear of W64A $\times s h 1^{*}$. The black arrow identified the $s h 1^{*}$ kernels, Bar $=0.5 \mathrm{~cm}$. b Comparison of osmolality in Z58 and sh ${ }^{*}$ kernels. c TLC analyses of total soluble sugars from Z58 and sh ${ }^{*}$ kernels. Fru, Glu and Suc represented fructose, glucose and sucrose, respectively, Mix was a mixture of them. The two groups of gels cropped from different parts of the same gel which is presented in additional Fig. S2 and the cropped areas are labelled using black borders. $\mathbf{d}$ Comparison of soluble sugar

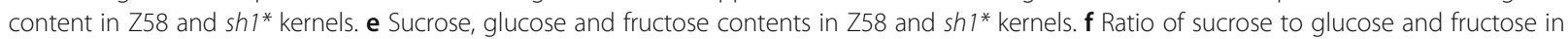
kernels of Z58 and sh $1^{*}$. All experiments mentioned in this picture were performed with three biological replicates and error bars represent SD

and 16 DAP, even more than 2.5 -fold soluble sugar content of that of Z58 kernels (Fig. 5c, d). These results were consistent with the differences of osmotic potential in kernels between $s h 1^{*}$ and Z58 (Fig. 5b). Therefore, the dramatical increase of total soluble sugars in developing kernels caused by SH1 mutation may be the main reason for the enhanced osmotic potential of $s h 1^{*}$ kernels. Moreover, the total soluble sugars content in Z58 kernels exhibited a continuous downward trend since 9 DAP, consistent with the rising starch content (Figs. 1j, 5d). However, the total soluble sugars content in $\operatorname{sh} 1 *$ kernels of $9,12,14$ and 16 DAP presented a sustained increase, consistent with slow starch accumulation compared to Z58 (Figs. 1j, 5d). While soluble sugars content in sh1* kernels was gradually reduced during 16 to 28 DAP, starch synthesis was markedly increased (Figs. 1j, 5d).
To further analyze the differences in soluble sugar components of kernels between $s h 1^{*}$ and Z58, the fructose, sucrose and glucose extracted from $s h 1^{*}$ and Z58 were assayed by thin layer chromatography (TLC; Fig. 5c). The contents of sucrose, fructose and glucose, and the ratios of sucrose to glucose and fructose in $s h 1^{*}$ kernels were significantly higher than that of Z58, except for the kernels of 9 DAP (Fig. 5e, f). Hence, $s h 1^{*}$ affected the balance of carbon metabolism and the distribution of carbohydrates in developing maize seeds, and resulted in significant changes in osmotic potential.

\section{sh 1* affected the transcription of genes involved in sugar metabolism and starch synthesis}

The expression of carbon metabolism related genes in endosperm of sh1*and Z58 at different developmental stages were detected by real-time quantitative 
PCR (qRT-PCR; Fig. 6). These genes included miniature seed1 (Mn1, GRMZM2G119689), hexokinase 2 (HxK2, GRMZM2G432801), fructokinase 2 (FRK2, GRMZM2G051677), phosphoglucomutase (PGM1, GRMZM2G023289), phosphohexose isomerase (PHI1, GRMZM2G065083), shrunken 2 (Sh2, GRMZM2G429899), brittle endosperm 2 (Bt2, GRMZM2G068506), dull endosperm 1 (Du1, GRMZM2G141399) and sugary 2 (Su2, GRMZM2G348551).

Sucrose degradation and utilization in endosperm mainly depends on two pathways. When functions of $S H 1$, the key gene of one of the two pathways, was lost, $M n 1$ encoding a key enzyme in another pathway may be up-regulated, as a result of feedback adjustment of sucrose concentration. To clarify this assumption, the expressions of $M n 1$ in endosperm of $s h 1^{*}$ and Z58 at different developmental stages were detected. These results showed that the expression level of $M n 1$ in $s h 1^{*}$ was significantly higher than that of Z58 at the same stage (Fig. 6a). As two mediators for coupling sucrose breakdown with hexose phosphate pool by Mn1-mediated pathway, $H x K 2$ and FRK2 had comparable or higher expression levels in sh1* mutant compared with Z58 (Fig. 6b, c). These not only helped to compensate for the loss of SH1mediated sucrose degradation pathway in sh1*, but also contributed to the accumulation of hexose phosphate pools, the main precursors for downstream carbon metabolic pathways. As the three main components of the hexose phosphate pools, the conversions of Glc-6-P, Fru-6-P and Glc-1-P are mainly coordinated by PHI1 and PGM1. Compared with Z58, the expression level of PHI1 was significantly upregulated in $s h 1 *$, while $P G M 1$ dramatically down-regulated (Fig. 6d, e). PHI1 is responsible for catalyzing the conversion between Glc-6-P and Fru-6-P. The up-regulated expression of PHI1 in sh1* facilitated the coordinated transformation between Glc-6-P and Fru-6-P. PGM1 catalyzes the transformation between glucose phosphate isomers, and its

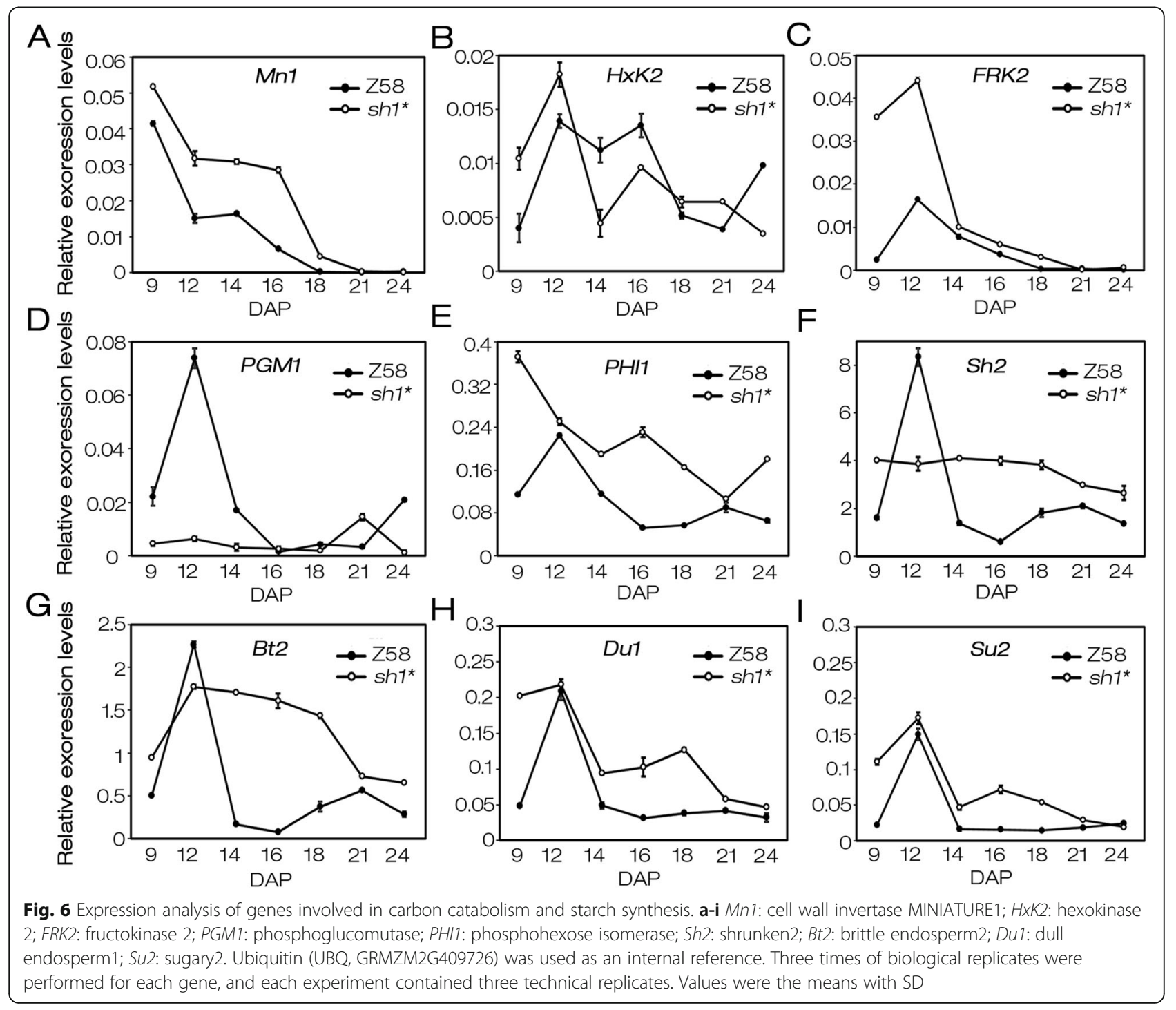


dramatic down-regulation will be unfavorable to the conversion Glc-6-P to Glc-1-P (a direct precursor for starch synthesis). The lack of Glc-1-P may induce the expression of starch synthesis-related genes, as a result of feedback regulation. To clarify this assumption, expression levels of several key genes involved in starch synthesis, including Sh2, Bt2, Du1 and Su2, were detected in kernels of $s h 1^{*}$ and Z58. These results displayed that, compared with Z58, the expression levels of $S h 2, B t 2, D u 1$ and $S u 2$ were obviously up-regulated during kernels development of $s h 1^{*}$, except for $S h 2$ and $B t 2$ at 12 DAP (Fig. 6f-g). Therefore, the decrease of starch content in sh1* might be attributed to the lack of substrate for starch synthesis caused by the loss of SH1-mediated sucrose degradation pathway.

\section{The kernels in $s h 1 *$ had a significantly reduced Glc-1-P content}

The contents of Glc-1-P and Glc-6-P were detected in the 12 DAP kernels of Z58 and sh1* (Fig. 7). The results showed that the contents of Glc-6-P was almost the same between $s h 1^{*}$ and Z58 (Fig. 7a-c), whereas compared with $\mathrm{Z} 58$, the content of Glc-1-P was greatly reduced in $\operatorname{sh} 1 *$ (Fig. 7d-f). The content of Glc-1-P in kernels of $s h 1^{*}$ was $17 \mu \mathrm{g} \mathrm{g}^{-1} \mathrm{FW}$, which was only about $5.2 \%$ of that in Z58 (326 $\left.\mu \mathrm{g} \mathrm{g}^{-1} \mathrm{FW}\right)$, indicating that the substrates deficiency may be an important factor of the decreased starch content in $s h 1^{*}$. Therefore, the functional loss of SH1 led to more carbon flow to the glycolysis pathway, and less to starch synthesis pathways, resulting in shrunken phenotype of $s h 1^{*}$ kernels (Fig. 1a, b).

\section{Discussion}

As the main form of carbohydrates transported from vegetative tissues to the reproductive organs, sucrose are vital for carbon source supply, energy metabolism, synthesis of cellulose and starch and so on. In addition, sucrose and its degradation products (hexoses) also function as signaling molecules to regulate cell fate determination and kernel development by affecting the expression of related genes [2, 29-31]. Hence, the regular sucrose catabolic pathways

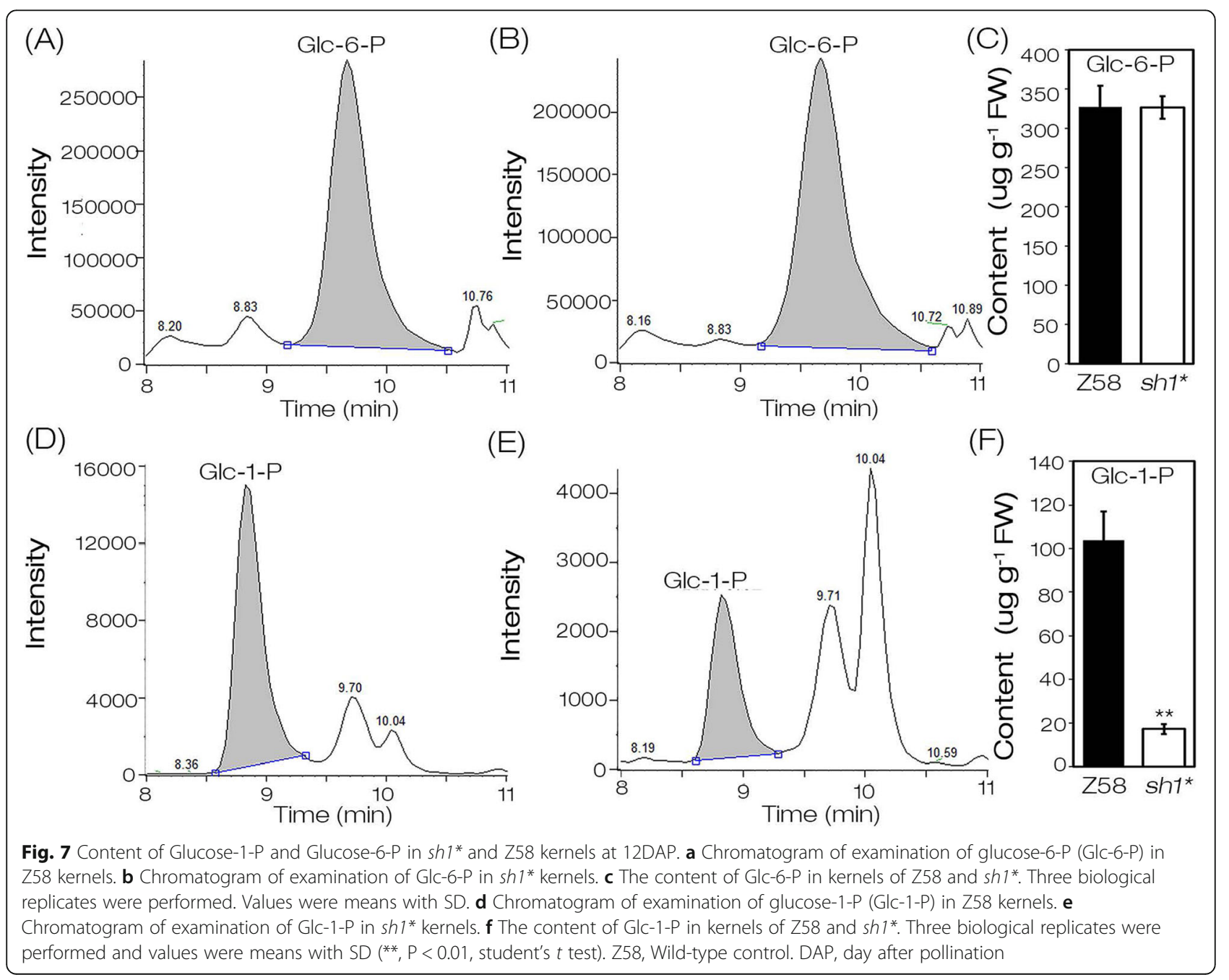


are essential for normal kernel development. Because of its importance, $\mathrm{SH} 1$ has attracted attentions as a key enzyme for sucrose degradation. The early studies of $\mathrm{SH} 1$ mostly focused on the gene structure [32,33], the enzyme activity [28, 34, 35], and the interallelic complementation at the $S h$ locus [36]. Recent research has shown that the functional loss of $\mathrm{SH} 1$ led to the decrease of starch accumulation $[8,11]$. However, the molecular and physiological regulatory mechanisms are still unclear. In this study, a new allelic mutant $\left(s h 1^{*}\right)$ of $\operatorname{sh} 1$ was identified and characterized. The mutation of SH1 in sh1* caused more carbon sources to exist as soluble sugars, and less flow to starch synthesis pathway (Figs. 1j, 5d, 7f). The modulated carbohydrates distribution led to the lack of starchsynthesis substrates and the high osmotic stress in developing endosperm cells in $s h 1^{*}$.

\section{The significant decrease of G-1-P content caused by the $\mathrm{SH} 1$ null mutation contributed greatly to the reduction of starch synthesis}

Previous studies showed that the functional loss of SH1 led to significant decrease in the starch content and shrunken phenotype of kernels in maize. Chourey et al. (1998) reported that the starch contents in kernels of sh1 was about 78\% of that in WT [8]. Guan et al. (2017) observed the starch content in sh1-m, a new allelic mutant of $S H 1$, was about $62 \%$ of that in WT, but thereof is no any explanation for this result [11]. In this study, time-course detection displayed that the starch in kernels of $s h 1 *$ were dramatically less accumulated at same stages, except for the kernels at 9 DAP (Fig. 1j). Hence, it is confirmed that $S H 1$ plays an important role in regulating starch synthesis.

The expression levels of key genes (such as Sh2 and $B t 2)$ related to starch synthesis indicated that they were significantly up-regulated in sh1* (Fig. 6f-i), suggesting the ability of starch synthesis in sh1* may not be weakened. Giroux et al. (1994) reported that the expression of storage product genes, specifically Bt2 and Sh2 encoding ADP-glucose pyrophosphorylase subunits, could be induced by elevated sucrose levels [45]. So, the increased expression of starch synthesis related genes may be attributed to the elevated sucrose levels in the $s h 1^{*}$ mutant (Fig. 5c, e). These results suggested that the decrease in starch content of $s h 1^{*}$ kernels might be not due to the weakened starch synthesis ability.

G-1-P, the direct substrate for starch synthesis, is mainly transformed from the two branches of UDP-Glc and G-6-P. UDP-Glc, as one of the sucrose degradation products catalyzed by SUS, the significant decrease of SUS activity inevitably reduces the production of UDPGlc, which in turn leads to the reduction of G-1-P converted from UDP-Glc. Although the SUS family has 20 members in the maize genome (Fig. 3a), the loss-of- function of $\mathrm{SH} 1$ leads to the decrease of approximately $90-95 \%$ of SUS activity in maize endosperm $[28,34,36]$, indicating that $\mathrm{SH} 1$ plays a key role in sucrose degradation of maize endosperm cells. In the study, a single base mutation of $\mathrm{SH} 1$ results in the loss of about $92 \%$ of SUS activity in sh1* endosperm (Fig. 2e) and shrunken phenotype (Fig. 1a and b), so sh1* can be considered as a mutant with loss of $\mathrm{SH} 1$ function. Therefore, G-1-P converted from UDP-Glc will be greatly reduced in endosperm of $s h 1 *$ mutant. In addition, the conversion of G-6$\mathrm{P}$ to G-1-P requires PGM1 catalysis. The null mutation of SH1 resulted in a significant down-regulation of PGM1 expression (Fig. 6d), suggesting a rate-limiting flux of G-6$\mathrm{P}$ to G-1-P. Indeed, the content of Glc-1-P in endosperm of $\operatorname{sh} 1 *\left(17 \mu \mathrm{g} \mathrm{g}^{-1}\right.$ fresh weigh) was only $5.2 \%$ of Z58 (326 $\mu \mathrm{g} \mathrm{g}^{-1}$ fresh weigh; Fig. $\left.7 \mathrm{~d}-\mathrm{f}\right)$. The results indicated that the loss-of-function of $\mathrm{SH} 1$ largely blocked the production of G-1-P, suggesting that SH1-encoded sucrose synthase may play critical roles in the supply of substrates for starch synthesis during kernel development. Therefore, the SH1-mediated sucrose degradation greatly contributed to kernels development and starch synthesis by regulating the flow of carbohydrates (Fig. 8).

\section{The lack of SH1-mediated sucrose degradation triggered transcriptional responses distinct from that mediated by MN1}

Two key enzyme families, INVs and SUSs are involved in sucrose degradation. INVs irreversibly catalyze the breakdown of sucrose into fructose and glucose, whereas SUSs catalyze the conversion of sucrose to fructose and UDP-Glc in a reversible manner [9, 25-27]. In maize, $\mathrm{Mn} 1$ and SH1 are widely considered as key enzymes in the INVs and SUSs family, respectively. Both loss-offunction mutants of $M n 1$ and SH1 ( $m n 1$ and sh1) had significant kernel development defects and reduced starch content [8, 11, 24, 27]. However, the regulation mechanisms of kernel developmental defects caused by mutation of $\mathrm{Mn1}$ or SH1 are significantly different. Chourey et al. (2012) found that $M n 1$ mutation resulted in an obvious reduction in fructose and glucose contents, and a significantly reduced ratio of hexose to sucrose [24]. Our results showed that although the functional loss of $S H 1$ led to an obviously reduced ratio of hexose to sucrose, the contents of fructose, glucose and total soluble sugars in $s h 1$ * were significantly higher than that of WT (Fig. 5e, f). Correspondingly, a large number of genes related to carbon metabolism and starch synthesis had significantly differential expression trends between $m n 1$ and sh1. They thought that the hexose-deficiency was the main cause of the metabolic coordinated regulation in $m n 1$ [24]. In this study, we found that almost all genes involved in carbon metabolism and starch synthesis, including $M n 1, S u 2, F R K 2$, 


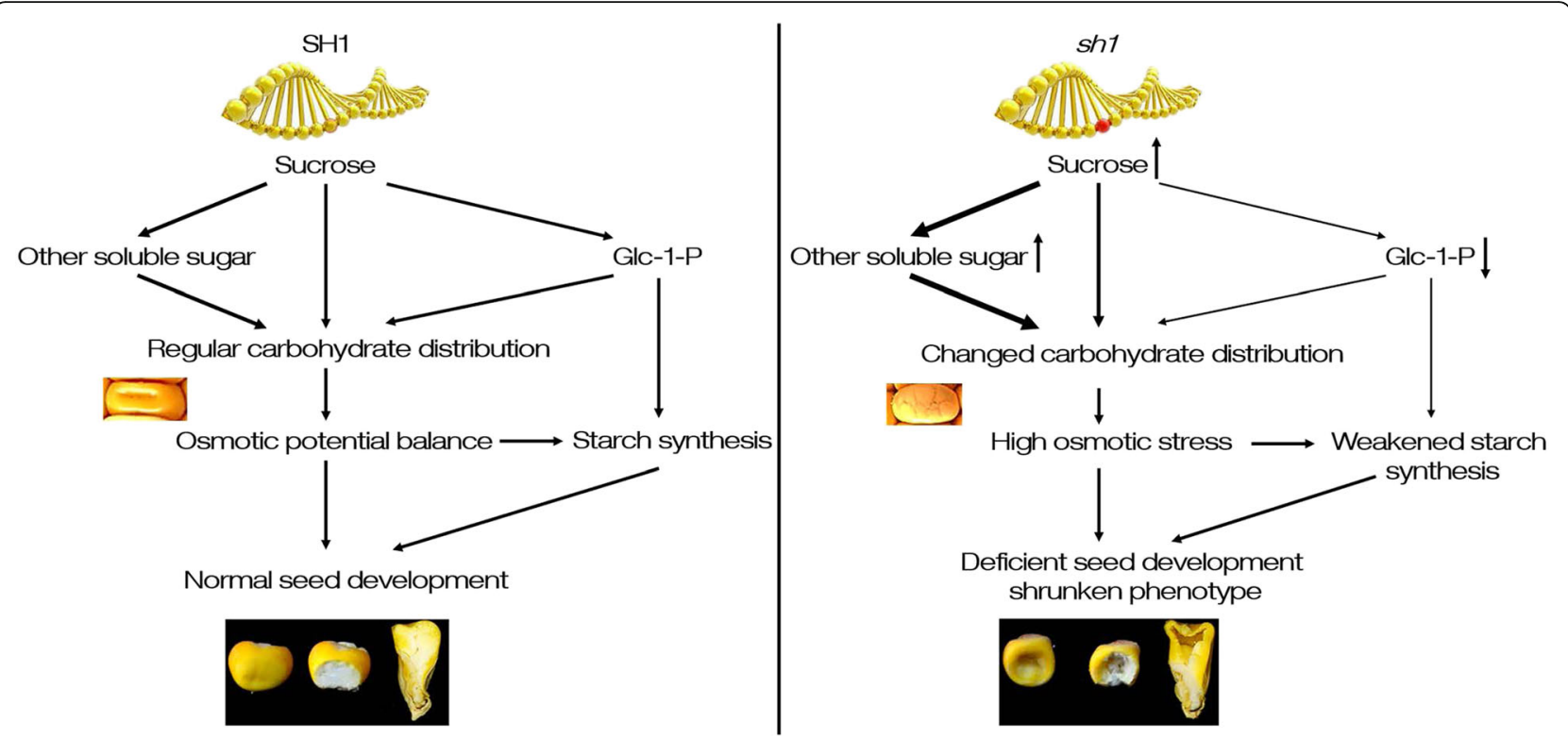

Fig. 8 Model of the influence of carbohydrate flow mediated by $\mathrm{SH} 1$ on kernel development. The thickness of the arrow indicated the activity of the reaction. Upward arrows behind sucrose and other soluble sugar indicated the increased content, downward arrows behind Glc-1-P indicated decreased content in the case of $\mathrm{SH} 1$ mutation

PHI1, Sh2, Bt2 and Du1, were significantly up-regulated in the endosperm of sh1* (Fig. 6). While SH1-mediated sucrose degradation pathway was blocked in the $\operatorname{sh} 1 *$, the Mn1-mediated sucrose degradation pathway was significantly induced to compensate for the sucrose degradation defects caused by $S H 1$ mutation. It is noticeable that when Mn1-mediated sucrose degradation was inhibited in the mn1 mutant, SH1-mediated sucrose degradation would be significantly inhibited [24]. Based on these results, we suggested that $M n 1$ and $S H 1$ might function in different ways during kernel development in maize.

To explore the roles of sucrose degradation and utilization during maize kernel development more clearly and accurately, more efforts are still required. For example, generation of constructing sh $1 \mathrm{mn} 1$ mutants by crossing $s h 1^{*}$ and $m n 1$ may help to understand the functional interactions between INVs and SUSs in details.

\section{Conclusions}

In the study, a novel SH1 mutant (sh1*) was characterized. Null mutation of $\mathrm{SH} 1$ resulted in defects in kernel development and starch granule formation, including markedly decreased starch content, significantly increased soluble sugar content and osmotic potential. The measurement results of phosphorylated sugars showed that the loss-of-function of $S H 1$ resulted in a significant decrease of G-1-P content in sh1* endosperm. As a direct source of starch synthesis, the decrease of Glc-1-P may result in a significant reduction in carbohydrates that flow to starch synthesis, ultimately contributing to the defects in starch granule development and reduction of starch content. These results provided new insights into understanding of the roles of SH1 in starch synthesis and maize kernel development.

\section{Methods}

\section{Plant materials}

The $s h 1 *$ mutant was separated from EMS induced mutants' library in Z58 genetic background in our laboratory. The inbred line Z58 is the female parent of the hybrid Zhengdan958 and has been widely used in the maize hybrid breeding in china. The uFmu-02854 mutant was obtained from the Maize Genetics Cooperation stock center. The $s h 1^{*}$ was crossed to W64A to produce F1, and F1 self-crossing generates F2 mapping population. All plants were cultivated in the field on the outskirts of Jinan, Shandong Province, China. Kernels were harvested at 9, 12, 14, 16, 18, 21, 24 and 28 DAP.

\section{Paraffin sections}

For anatomical observation, kernels were sectioned based on paraffin embedding as previously described [3]. Briefly, kernels were fixed in FAA buffer (glacial acetic acid: formaldehyde: $50 \%$ ethanol, $1: 1: 18$ ) at $4{ }^{\circ} \mathrm{C}$ for 24 $\mathrm{h}$, and dehydrated in different concentrations of ethanol. Subsequently, the samples were embedded in paraffin, cut into $8 \mu \mathrm{m}$-thick slices, and stained with basic fuchsin and observed using an Olympus SZ61 microscope.

\section{Scanning Electron microscopy}

For starch granule observation, the naturally ruptured endosperm from dry mature kernels were coated with 
gold and then observed using scanning electron microscopy (FEI Quanta250 FEG).

\section{Map-based cloning}

To map the sh1* locus, 2400 individuals from a W64A $\times$ sh1* F2 segregating population were used. Preliminary mapping was performed using molecular markers distributed on maize chromosome 9. The molecular markers listed in Additional file 1 (Table S1) were used for finemapping and eventually the $s h 1^{*}$ locus was localized to a $97.12 \mathrm{~kb}$ region.

The genomic DNA of sh1* and Z58 were extracted. The nucleotide sequence of predicted genes within this region was amplified from the $s h 1^{*}$ and Z58 genomic DNA using TransStart FastPfu DNA polymerase (Transgen Biotech), and aligned after sequencing by Shanghai Boshang Biotechnology Co., Ltd.

\section{Phylogenetic analysis of SUSs}

The amino acid sequences of SUSs were download from MaizeGDB (https://maizegdb.org/). The phylogenetic analysis of them were performed with the maximum likelihood according to [7]. Phylogenetic tree was constructed using MEGA7.0 Amino acid sequences alignment of GRMZM2G089713 (SH1), GRMZM2G152980 (SUS1) and GRMZM2G318780 (SUS2) were performed using DNAMAN 7.0 (Lynnon Corporation, USA).

\section{Subcellular localization}

The CDS sequence of $S H 1$ was ligated into pEarleyGate 103 using the Gateway TOPO cloning kit to generate a 35S::SH1: GFP fusion construct. Then, this vector was introduced into 6-weeks-old Nicotiana benthamiana leaves by Agrobacterium impregnation. After $48 \mathrm{~h}$, the Nicotiana benthamiana epidermis was examined with a confocal microscope (Zeiss LSM700). For protoplast transformation, the plasmid of 35S:: SH1:GFP was introduced into protoplast isolated from 2weeks-old Nicotiana benthamiana leaves via PEG-mediated transformation. After $18 \mathrm{~h}$, protoplasts were examined as described above. CD3-1001 (CFP) was used as a membrane protein marker and mixed with bacterial solution 1:1 to infect tobacco leaves and protoplast.

\section{The determination of osmolality}

The $\operatorname{sh} 1^{*}$ and Z58 kernels at different developmental stages were homogenized and centrifuged separately, then pipetting $20 \mu \mathrm{l}$ of supernatant to use for the determination of osmolality by the Fiske Micro-Osmometer (Model 210). Three biological replicates were done.

\section{The measurement of soluble sugar and starch content}

The total soluble sugars of $s h 1^{*}$ and Z58 kernels at different developmental stages were extracted three times in $80 \%$ ethanol at $80^{\circ} \mathrm{C}$ for $30 \mathrm{~min}$ after thoroughly triturated, respectively. For the quantification of soluble sugars, a constant volume of those supernatants were maintain in the wake of $80 \%$ ethanol added, then extracts were diluted in distilled water and measured with the anthranone-sulfuric acid reagent at $620 \mathrm{~nm}$ as described previously $[37,38]$. The fructose, glucose and sucrose were differentiated by thin layer chromatography (TLC) based on the method by Li et al. (2008) [20]. The total soluble sugars extracted from kernels in same fresh weight $(0.2 \mathrm{mg})$ were used for TLC analysis. TLC image were further analyzed at $390 \mathrm{~nm}$ wavelength using a Cs930 dual-wavelength chromatoscanner (Shimadzu) to semi-quantitated the content of sucrose, glucose and fructose. The pellets derived from total soluble sugars extraction were used for starch measurement. After removing the ethanol, those pellets were boiled with distilled water for $10 \mathrm{~min}$, then cooled down to room temperature, and decomposed those samples with $\mathrm{HClO}_{4}$ for $15 \mathrm{~min}$. The products of hydrolyzation were examined at $620 \mathrm{~nm}$ as described above. Formula below was for starch content calculation: Starch content $(\%)=$ $\mathrm{G} \times 0.9 \mathrm{FW}^{-1} \times 100 \%$. (G: content of glucose in these samples, FW: fresh weight of these kernels).

\section{RNA extraction and gene expression assays}

The $s h 1^{*}$ and Z58 kernels at different developmental stages were carefully removed the seed coat and separated the embryo and endosperm. The total RNA of embryos and endosperms were extracted using Trizol reagent (Sangon, China) as product manual. The $2 \mu \mathrm{g}$ total RNA of each sample was used as the template of cDNA synthesis that was carried out using a PrimeScript TM RT reagent Kit with genome DNA Eraser (Takara, China). SYBR Green PCR master mix kit (Takara, China) and Light Cycler 96 PCR machine (Roche) were used for real-time quantitative PCR (qRT-PCR) examination. Ubiquitin (UBQ, GRMZM2G409726) was used as an internal reference. Primers used in qRT-PCR were listed in Additional file 2 (Table S2). Three biological replicates were done for gene expression analysis.

\section{The measurement of Glc-1-P and Glc-6-P contents}

Glc-1-P and Glc-6-P contents were determined by the Wuhan Greensword Creation Technology Co. Ltd., using UHPLC-MS/MS according to the previous method [39]. Twelve DAP kernels of Z58 and sh 1 * were ground to power in the presence of liquid nitrogen. $5 \mu \mathrm{l}(25 \mathrm{ng})$ $\left[{ }^{13} \mathrm{C}_{6}\right]$ G-6-P at $5 \mu \mathrm{g} \mathrm{ml}^{-1}$ was added to the samples as an internal reference (I.S.) for accurate quantification. $250 \mu \mathrm{l}$ ice-cold mixture of chloroform and acetonitrile (3:7, $\left.\mathrm{v} \mathrm{v}^{-1}\right)$, was added to Z58 and sh1* samples, respectively. After thoroughly mixing, these samples were incubated at $-20^{\circ} \mathrm{C}$, with intermittent mixing, for $2 \mathrm{~h}$. Next, $200 \mu \mathrm{l}$ cold water in ice bath was added to the reaction 
solution, then vortexed for $3 \mathrm{~min}$. The upper aqueous acetonitrile phase was separated and transferred to new $1.5 \mathrm{ml}$ centrifuge tubes after centrifugation $(10,000 \times \mathrm{g}$, $4{ }^{\circ} \mathrm{C}$ ) for $5 \mathrm{~min}$. The chloroform phase was extracted once more and mixed with the aqueous acetonitrile phases. The residue, produced by evaporating the aqueous acetonitrile phases under mild nitrogen stream, was re-dissolved in $200 \mu \mathrm{l}$ borate buffer $(50 \mathrm{mM}, \mathrm{pH} 6.8)$, and purified with C18 SPE cartridge (50 mg of C18 sorbent), then washed with the borate buffer $(50 \mathrm{mM}, \mathrm{pH} 6.8$, $100 \mu \mathrm{l})$. The elutes were collected, and added with $180 \mu \mathrm{l}$ of 8 -DMQ solution $(0.014 \mathrm{M})$ into it. The reaction was carried out during the vigorously shaking at $25^{\circ} \mathrm{C}$ for 40 $\min$ and centrifuged $3 \mathrm{~min}$ at $10,000 \times \mathrm{g}$. The solution $(2.5 \mu \mathrm{l})$ was analyzed by ultra-high performance liquid chromatography-electron spray ionization-tandem mass spectrometry (UHPLC-ESI-MS/MS).

\section{The measurement of sucrose synthase activity}

A mixture of equal weight of endosperm at 16 DAP and chilled $0.01 \mathrm{U}$ tris-maleate buffer ( $\mathrm{pH} 7.0)$ was homogenized, and then centrifuge at 30,000 g for $20 \mathrm{~min}$. The supernatants were used as the enzyme sources for the routine assays. The activity of sucrose synthase was assayed according to the description by Tsai et al. (1970) [40]. The $0.55 \mathrm{ml}$ reaction mixture contained $30 \mu \mathrm{mol}$ of HEPES buffer (pH 8.0), $2.5 \mu \mathrm{mol} \mathrm{MgSO}_{4}, 2 \mu$ mol fructose, $1 \mu \mathrm{mol} \mathrm{UDP}$-glucose, and $10 \mu \mathrm{l}$ crude preparation. After the reaction at $30^{\circ} \mathrm{C}$ for $30 \mathrm{~min}, \mathrm{NaOH}$ was added to stop the reaction. Boiling water for $10 \mathrm{~min}$, cooling and adding $1.5 \mathrm{ml}$ of $30 \%$ hydrochloric acid and $0.5 \mathrm{ml}$ of $0.1 \%$ phloroglucinol, after shaking for $10 \mathrm{~min}$, the content of sucrose was determined at $480 \mathrm{~nm}$ wavelength after cooling, the standard curve of sucrose was made at the same time. Finally, the activity of sucrose synthase in the sample was calculated. Three biological replicates were done.

\section{Supplementary information}

Supplementary information accompanies this paper at https://doi.org/10. 1186/s12870-020-02478-1.

Additional file 1: Table S1. The list of molecular markers and primers used in gene cloning.

Additional file 2: Table S2. The list of primers used in $\mathrm{QRT}-\mathrm{PCR}$ reaction.

Additional file 3: Fig. S1. The sequencing data of GRMZM2G089713 in Z58 and sh $^{*}$.

Additional file 4: Fig. S2. Full size, unedited gel used for Fig. $5 c$ in the main text.

\section{Abbreviations}

Bt2: brittle endosperm 2; BETL: Basal endosperm transfer layer; DAP: Days after pollination; Du1: Dull endosperm 1; EMS: Ethyl methanesulfonate; FRK2: Fructokinase 2; Fru-6-P: Fructose-6-phosphate; Glc-1-P: Glucose-1phosphate; Glc-6-P: Glucose-6-phosphate; HXK2: Hexokinase 2; INV: Invertase; Mn1: MINIATURE1; ORF: Open reading frame; PGM1: Phosphoglucomutase; PHI1: Phosphohexose isomerase; qRT-PCR: Real-time quantitative PCR;
Sh2: Shrunken 2; Suz: Sugary 2; SUS: Sucrose synthase; SUTs: Sucrose transporters; TLC: Thin layer chromatography; UDP-Glc: Uridinediphosphoglucose; WT: Wild-type.

\section{Acknowledgements}

Not applicable.

\section{Authors' contributions}

$\mathrm{KL}, \mathrm{FW}, \mathrm{KWZ}$ and ZD conceived and designed the research; KZ, LG and WC performed most of the experiments; $\mathrm{BL}$ in protein localization; $\mathrm{WL}$ participated in the map-based cloning of $\mathrm{SH} 1 ; \mathrm{KL}$ contributed reagents/materials/analysis tools; $\mathrm{KL}, \mathrm{KZ}, \mathrm{CX}$ and $\mathrm{XZ}$ analyzed the data and wrote the paper. All authors have read and approved the manuscript.

\section{Funding}

This work was supported by grants from National Key R\&D Program of China (2016YFD0102104 and 2016YFE0200300); Shandong Provincial Natural Science Foundation, China (ZR2017YL011 and ZR2016CM05); and Shandong Provincial key research and development program, China (2017GSF21107). These funding bodies had no role in the design of the study and collection, analysis, and interpretation of data and in writing the manuscript.

\section{Availability of data and materials}

All data generated or analysed during this study are included in this published article and its additional information files. The sequencing data of GRMZM2G089713 in Z58 and sh1* see additional Fig. S1. All plant materials were obtained from Shandong University, Qingdao, China.

Ethics approval and consent to participate

Not applicable.

\section{Consent for publication}

Not applicable.

\section{Competing interests}

The authors declare that they have no competing interests.

\section{Author details}

${ }^{1}$ The Key Laboratory of Plant Development and Environment Adaptation Biology, Ministry of Education, School of Life Science, Shandong University, Qingdao 266237, China. ${ }^{2}$ Maize Institute of Shandong Academy of Agricultural Sciences, Jinan, Shandong, China. ${ }^{3}$ National Key Laboratory of Plant Molecular Genetics, CAS Center for Excellence in Molecular Plant Sciences, Institute of Plant Physiology and Ecology, Shanghai Institutes for Biological Sciences, Chinese Academy of Sciences, Shanghai 200032, China. ${ }^{4}$ School of Life Sciences, Southwest University, Chongqing 400715, China.

${ }^{5}$ State Key Laboratory of Crop Biology, College of Life Sciences, Shandong Agricultural University, Taian 271018, Shandong, China.

Received: 19 December 2019 Accepted: 1 June 2020 Published online: 08 June 2020

\section{References}

1. Sosso D, Luo D, Li QB, Sasse J, Yang J, Gendrot G. Seed filling in domesticated maize and rice depends on SWEET-mediated hexose transport. Nat Genet. 2015:47:1489-93.

2. Doll NM, Depege-Fargeix N, Rogowsky PM, Widiez T. Signaling in early maize kernel development. Mol Plant. 2017;10:375-88.

3. Xie G, Li Z, Ran Q, Wang H, Zhang J. Over-expression of mutated ZmDA1 or ZmDAR1 gene improves maize kernel yield by enhancing starch synthesis. Plant Biotechnol J. 2018;16:234-44.

4. Baker RF, Leach KA, Braun DM. SWEET as sugar: new sucrose effluxers in plants. Mol Plant. 2012;5:766-8.

5. Braun DM. SWEET! The pathway is complete. Plant Sci. 2012;335:173-4.

6. Bihmidine S, Hunter CT III, Johns CE, Koch KE, Braun DM. Regulation of assimilate import into sink organs: update on molecular drivers of sink strength. Front Plant Sci. 2013;4:177.

7. Eom JS, Chen LQ, Sosso D, Julius BT, Lin IW, Qu XQ, et al. SWEETs, transporters for intracellular and intercellular sugar translocation. Curr Opin Plant Biol. 2015;25:53-62. 
8. Chourey PS, Taliercio EW, Carlson SJ, Ruan YL. Genetic evidence that the two isozymes of sucrose synthase present in developing maize endosperm are critical, one for cell wall integrity and the other for starch biosynthesis. Mol Gen Genet MGG. 1998:259:88-96.

9. Subbaiah CC, Sachs MM. Altered patterns of sucrose synthase phosphorylation and localization precede callose induction and root tip death in anoxic maize seedlings. Plant Physiol. 2001;125:585-94.

10. Bahaji A, Li J, Sánchez-López ÁM, Baroja-Fernández E, Muñoz FJ, Ovecka M et al. Starch biosynthesis, its regulation and biotechnological approaches to improve crop yields. Biotechnol Adv. 2014;32:87-106.

11. Guan H, Dong Y, Liu C, He C, Liu C, Liu Q, et al. A splice site mutation in shrunken1-m causes the shrunken 1 mutant phenotype in maize. Plant Growth Regul. 2017;83:429-39.

12. Sakr S, Wang M, Dedaldechamp F, Perez-Garcia MD, Oge L, Hamama L, et al. The sugar-signaling hub: overview of regulators and interaction with the hormonal and metabolic network. Int J Mol Sci. 2018;19:E2506.

13. Qu J, Ma C, Feng J, Xu S, Wang L, Li F. Transcriptome dynamics during maize endosperm development. PLoS One. 2016;11:e0163814.

14. Yao D, Qi W, Li X, Yang Q, Yan S, Ling H, et al. Maize opaque10 encodes a cereal-specific protein that is essential for the proper distribution of zeins in endosperm protein dodies. PLoS Genet. 2016;12:e1006270.

15. Kang BH, Xiong Y, Williams DS, Pozueta-Romero D, Chourey PS. Miniature1encoded cell wall invertase is essential for assembly and function of wall-ingrowth in the maize endosperm transfer cell. Plant Physiol. 2009;151:1366-76.

16. Ruan YL. Signaling role of sucrose metabolism in development. Mol Plant. 2012;5:763-5.

17. Angeles-Nunez JG, Tiessen A. Arabidopsis sucrose synthase 2 and 3 modulate metabolic homeostasis and direct carbon towards starch synthesis in developing seeds. Planta. 2010;232:701-18.

18. Xu SM, Brill E, Llewellyn DJ, Furbank RT, Ruan YL. Overexpression of a potato sucrose synthase gene in cotton accelerates leaf expansion, reduces seed abortion, and enhances fiber production. Mol Plant. 2012;5:430-41.

19. Braun DM, Wang $L$, Ruan $Y L$. Understanding and manipulating sucrose phloem loading, unloading, metabolism, and signalling to enhance crop yield and food security. J Exp Bot. 2013;65:1713-35.

20. Li K, Xu C, Li Z, Zhang K, Yang A, Zhang J. Comparative proteome analyses of phosphorus responses in maize (Zea mays L.) roots of wild-type and a low-P-tolerant mutant reveal root characteristic associated with phosphorus efficiency. Plant J. 2008;55:927-39.

21. Juarez-Colunga S, Lopez-Gonzalez C, Morales-Elias NC, Massange-Sanchez JA, Trachsel S, Tiessen A. Genome-wide analysis of the invertase gene family from maize. Plant Mol Biol. 2018:97:385-406.

22. Vilhar B, Kladnik A, Blejec A, Chourey PS, Dermastia M. Cytometrical evidence that the loss of seed weight in the miniature1 seed mutant of maize is associated with reduced mitotic activity in the developing endosperm. Plant Physiol. 2002;129:23-30.

23. Chourey PS, Li QB, Kumar D. Sugar-hormone cross-talk in seed development: two redundant pathways of IAA biosynthesis are regulated differentially in the invertase-deficient miniature 1 ( $\mathrm{mn} 1$ ) seed mutant in maize. Mol Plant. 2010;3:1026-36.

24. Chourey PS, Li QB, Cevallos-Cevallos J. Pleiotropy and its dissection through a metabolic gene Miniature1 (Mn1) that encodes a cell wall invertase in developing seeds of maize. Plant Sci. 2012;184:45-53.

25. Jiang SY, Chi YH, Wang JZ, Zhou JX, Cheng YS, Zhang BL, et al. Sucrose metabolism gene families and their biological functions. Sci Rep-UK. 2015;5:17583.

26. Duncan KA, Hardin SC, Huber SC. The three maize sucrose synthase isoforms differ in distribution, localization, and phosphorylation. Plant Cell Physiol. 2006:47:959-71.

27. Ren X, Zhang J. Research progresses on the key enzymes involved in sucrose metabolism in maize. Carbohydr Res. 2013;368:29-34.

28. Chourey PS, Nelson OE. The enzymatic deficiency conditioned by the shrunken-1 mutations in maize. Biochem Genet. 1976;14:1041-55.

29. Gupta AK, Kaur N. Sugar signalling and gene expression in relation to carbohydrate metabolism under abiotic stresses in plants. J Biosci. 2005:30:761-76.

30. Ruan YL. Sucrose metabolism: gateway to diverse carbon use and sugar signaling. Ann Rev Plant Biol. 2014;65:33-67.

31. Ren XD, Liu HM, Liu YH, Hu YF, Zhang JJ, Huang YB. Influence of sugars and hormones on the genes involved in sucrose metabolism in maize endosperms. Genet Mol Res. 2015;14:1671-8.

32. Werr W, Frommer WB, Maas C, Starlinger P. Structure of the sucrose synthase gene on chromosome 9 of Zea mays L. EMBO J. 1985:4:1373-80.
33. Shaw JR, Ferl RJ, Baier J, St Clair D, Carson C, McCarty DR, et al. Structural features of the maize sus1 gene and protein. Plant Physiol. 1994;106:1659-65..

34. Chourey PS, Nelson OE. Studies on sucrose synthetase in the developing endosperm and embryos of normal and shrunken genotypes. Maize Genetics Cooperation News Letter. 1978;52:125-7.

35. Cobb BG, Hannah LC. Shrunken-1 encoded sucrose synthase is not required for sucrose synthesis in the maize endosperm. Plant Physiol. 1988;88:1219-21.

36. Chourey PS, Nelson OE. Interallelic complementation at the sh locus in maize at the enzyme level. Genetics. 1979;91:317-25.

37. Yemm EW, Willis AJ. The estimation of carbohydrates in plant extracts by anthrone. Biochem J. 1954:57:508-14.

38. Li N, Zhang S, Zhao Y, Li B, Zhang J. Over-expression of AGPase genes enhances seed weight and starch content in transgenic maize. Planta. 2011 233:241-50

39. Luo XT, Cai BD, Jiang HP, Xiao HM, Yuan BF, Feng YQ. Sensitive analysis of trehalose-6-phosphate and related sugar phosphates in plant tissues by chemical derivatization combined with hydrophilic interaction liquid chromatographytandem mass spectrometry. J Chromatogr A. 2019;1592:82-90.

40. Tsai CY, Salamini F, Nelson OE. Enzymes of carbohydrate metabolism in the developing endosperm of maize. Plant Physiol. 1970;46:299-306.

\section{Publisher's Note}

Springer Nature remains neutral with regard to jurisdictional claims in published maps and institutional affiliations.
Ready to submit your research? Choose BMC and benefit from:

- fast, convenient online submission

- thorough peer review by experienced researchers in your field

- rapid publication on acceptance

- support for research data, including large and complex data types

- gold Open Access which fosters wider collaboration and increased citations

- maximum visibility for your research: over $100 \mathrm{M}$ website views per year

At BMC, research is always in progress.

Learn more biomedcentral.com/submissions 